\title{
Synthesis and Characterization of a Layered Uranium Phosphites
}

\author{
Zhen Zhu \\ Institute of Nanostructured Materials \\ School of Materials Science and Engineering \\ And Tianjin Key Laboratory of Fiber Modification and Functional Fiber \\ Tianjin Polytechnic University \\ Tianjin 300160, China \\ E-mail: zhuzhen183@163.com
}

\begin{abstract}
A novel layered oxouranium phosphite open-structure $\left[\mathrm{C}_{10} \mathrm{H}_{24} \mathrm{~N}_{2}\right] \cdot\left[\left(\mathrm{UO}_{2}\right)_{2}\left(\mathrm{HPO}_{3}\right)_{3}\right] \cdot \mathrm{H}_{2} \mathrm{O}$ (denoted as ZZ-1) was hydrothermally synthesized by using isophorondiamine (IPDA) as the template. Single crystal structure refinement discloses that ZZ-1 crystallizes in the monoclinic space group P $2{ }_{1} \mathrm{c}$ with the cell parameters of a $=18.347(4) \AA, b=$ 6.7341(13) $\AA, c=20.639(4) \AA, \beta=114.61(3)^{\circ}, V=2318.2(8) \AA^{3}, Z=2$. The structure of $Z Z-1$ is constructed by $\mathrm{UO}_{7}$ monomer, $\mathrm{U}_{2} \mathrm{O}_{12}$ dimer and $\mathrm{HPO}_{3}$ groups. The monomeric $\mathrm{UO}_{7}$ or dimeric $\mathrm{U}_{2} \mathrm{O}_{12}$ is connected by $\mathrm{HPO}_{3}$ groups to form two types of 4-ring chains. An undulating sheet is then generated by connecting these two types of chains via $\mathrm{HPO}_{3}$ bridges. The layer structure is formed by stacking the sheet along the direction with the organoamine cations and water molecules intercalated in the interlayer spaces with strong H-bonds to the framework oxygen atoms. Although a mixture of cis and trans IPDA is used, only cis PDA serves as the template. A typical green light emission of $\mathrm{UO}_{2}{ }^{2+}$ is observed when excited by $266 \mathrm{~nm}$ laser.
\end{abstract}

Keywords: Hydrothermal synthesis, Open-framework compound, Single crystal structure, Template synthesis, Oxouranium phosphite

\section{Introduction}

Open-framework materials have been extensively pursued due to their structural diversities and potential applications in catalysis, aborption, separation,ion-exchange and host-guest chemistry. Recently, replacing tetrahedral $\mathrm{HOPO}_{3}{ }^{2-}$ by pseudo pyramidal $\mathrm{HPO}_{3}{ }^{2-}$ has been demonstrated to be an effective approach to generate more open framework structures. The occurrence of $\mathrm{HOPO}_{3}, \mathrm{O}=\mathrm{PO}_{3}$ groups in some large pore zeolitic compounds verified its possibility. From this view, replacing tetrahedral phosphate groups by pseudo-pyramidal phosphite units has been considerably explored and resulted in a new family of metal phosphite compounds in the past decade. Metal phosphites with novel structures and compositions have experienced dramatic expansion under the assistance of various organoamines. Crystals with extra-large pores were also achieved, including TJPU-3 with 20-ring apertures, ZnHPO-CJ1 and Cr-NKU-24 with 24-ring opennings, and NTHU-5 with the largest 26-ring micropores. As well-known, uranium is fruitful in coordination geometry and valence state, which lead to structural versitility of uranium-containing framework. However, open-framework uranium phosphites are seldom in the documents.

On the other hand, organic isomer separation and recognition have stimulated extensive research into the preparation of separation media. Inorganic matrix for isomer recognition have been considerably pursued but the occurrence is scarce. Succesful examples for isomer seperation include the ND-1 and a tin phosphate that only use trans-1,2-diaminocyclohexane as the template. Herein, by using isophorondiammine(IPDA) as the template, a layed oxouranium phosphite, $\left[\mathrm{C}_{10} \mathrm{H}_{24} \mathrm{~N}_{2}\right] \cdot\left[\left(\mathrm{UO}_{2}\right)_{2}\left(\mathrm{HPO}_{3}\right)_{3}\right] \cdot \mathrm{H}_{2} \mathrm{O}(\mathrm{ZZ}-1)$ was isolated.Only cis-IPDA served as template.

\section{Experimental}

ZZ-1 was hydrothermally synthesized by using isophorondiamine (mixtures of cis- and trans- isomers, Aldrich) as the template. All chemicals were used without purification.Typically, $0.107 \mathrm{~g} \mathrm{UO}_{2}\left(\mathrm{CH}_{3} \mathrm{CO}_{2}\right)_{2} \cdot 2 \mathrm{H}_{2} \mathrm{O}, 0.116 \mathrm{~g} \mathrm{H}_{3} \mathrm{PO}_{3}$ and $0.042 \mathrm{~g}$ IPDA were dispersed into $5 \mathrm{ml}$ distilled water. After stirred for $1.5 \mathrm{hs}$, the mixture was sealed in an autoclave and heated at $180^{\circ} \mathrm{C}$ for $144 \mathrm{hs}$. The hydrothermal reaction produced yellow stick-like crystals, which were washed by distilled water and dried at room temperature. The experimental and simulated XRD patterns are well matched, 
indicating the monophasic feature of the products. EDAX measurement gave the U:P ratio of 2:3.XPS gave the binding energy of $\mathrm{U} 4 \mathrm{f} 7 / 2$ of $381.2 \mathrm{eV}$ that very close to the value of $\mathrm{UO}_{3}$, indicating the hexavalent state of $\mathrm{U}$.

\section{Results and discussion}

ZZ-1 crystallizes in the monoclinic space group $\mathrm{P} 2_{1} \mathrm{c}$. The asymetric unit contains 31 non-hydrogen atoms(Figure 1A).The uranium atoms are all in pentagonal bipyramid geometry. Two vertex of the pentagonal bipyramid are occupied by acyl-oxygen atoms. While $\mathrm{U}(1)$ is a monomeric $\mathrm{UO}_{7}$, two adjacent $\mathrm{U}(2) \mathrm{O}_{7}$ groups are edge shared to form dimeric $\mathrm{U}_{2} \mathrm{O}_{12}$ (Figure 1B and 1C).All the phosphorous atoms are pseudo pyramidal $\mathrm{HPO}_{3}$ groups. A strong IR adsorption at $2378.1 \mathrm{~cm}^{-1}$ verifies the presence of P-H bonds.

\section{Insert Figure 1 Here \\ Insert Figure 2 Here}

The extended structure is built by $\mathrm{UO}_{7}, \mathrm{U}_{2} \mathrm{O}_{12}$, and $\mathrm{HPO}_{3}$ groups. The connection of these groups generate two types of 4-ring chains running along $\mathrm{b}$ axis. These two types of chains alternatively align along ab plane with a cut angle of about $104^{\circ}$. Connecting these two types of chains generates an undulating sheet that extended along ab plane. Stacking these sheets along $\mathrm{c}$ axis gives rise to a layer structure. The interlayer spaces are occupied by diprotonated IPDA cations and water molecules with complex H-bond to the framework oxygen. Interestingly, all the IDPA cations are in cis- form (Figure 1C).The means isolation ZZ-1 lead to the separation of cis-IPDA from the isomer mixtures.

UV light excited green light emission is a well-known character for uranyl-containing compounds, which is usually used to determine the presence of $\mathrm{U}(\mathrm{VI})$ in minerals. The fine structures of photoluminecence spectra of ZZ-1 show typical emission features upon irradiation with $266 \mathrm{~nm}$ UV light(Figure 2B).A strong peak at $525 \mathrm{~nm}$ companied by four weaker peaks at 504,549,575, and $604 \mathrm{~nm}$ is observed as a typical phenomenon for $\mathrm{UO}_{2}{ }^{2+}$

\section{Summary}

A new open-framework oxouranium phosphite, ZZ-1, has been synthesized under the hydrothermal condition by using isophorondiamine as the templates. It has a layered structure with the isophorondiamine cations and water molecules intercalated in the interlayer spaces. The connection of the $\mathrm{UO}_{6}$ pentagonal bipyramids and $\mathrm{HPO}_{3}$ pseudo pyramids generates two kinds of chain structures. These two types of chains fuse to form a 4,8-network sheet that are stacking to generate the layer structure. As well, separation of IPDA isomers is achieved by isolating ZZ-1 crystals. Fluorescent spectroscopy analysis reveals that the uranium phosphite exhibits interesting intense photoluminescence upon photoexcitation at $266 \mathrm{~nm}$.

\section{References}

A. J. Locock and P. C. Burns, J. (2004). Solid State Chem., 177, 2675.

C. L. Cahill, P. C. Burns. (2001). Inorg. Chem. 40, 1347.

Fernández, S., Mesa, J. L., Pizarro, J. L., Lezama, L., Arriortua, M. I., Rojo, T. Angew. (2002). Chem. Int. Ed. 41, 3683.

Harrison, W. T. A. (2006). Solid State Sci, 8, 371.

Huo, Q., Xu, R., Li, S., Ma, Z., Thomas, J. M., Jones, R. H.. Chippindale, A. M. (1992). Chem. Commun., 875.

J. A. Danis, W. H. Runde, B. Scott, J. Fettinger, B. Eichhorn. (2001). Chem. Commun., 2378.

J. Liang, J. Li, J. Yu, P. Chen, Q. Fang, F. Sun, R. Xu, Angew. (2006). Chem. Int. Chem, 45, 2546.

J. Xu, H. Li, Y. Cao, C. Huang, H. Zhang, D. Liu, Q. Yang, R. Sun. (2006). Chinese J. Struct. Chem., 11, 1380.

K. M. Ok, M. B. Doran, D. O Hare. (2007). Dalton Trans, 3325.

M.E. Davis. (2002). Nature, 417, 813.

S. Mandal, M. Chandra, S. Natarajan. (2007). Inorg. Chem., 46, 7935-7943.

Shi, Z., Li, G.., Zhang, D., Hua, J., \& Feng, S. (2003). Inorg. Chem., 42, 2357.

T. H. Bray, J. D. Gorden, T. E. Albrecht-Schmitt, J. (2008). Solid State Chem., 181, 2199.

X. Yuan, Y. Li, Y. Xu, X. You, R. J. Linhardt, Eur. J. (2007). Inorg. Chem., 33, 858.

Y. L. Lai, K. H. Lii, S. L. Wang, J. (2007). Am. Chem. Soc., 129, 5350.

Y. Yang, N. Li, H. Song, H. Wang, W. Chen, S. Xiang. (2007). Chem. Mater., 19, 1889.

Y. Yang, Y. Zhao, J. Yu, S. Wu, R. Wang. (2008). Inorg. Chem., 47, 769.

Y. Yang, Y. Zhao, J. Yu, Y. Zhou, N. Pang, H. Su, S. Pang, Z. (2008). Anorg. Allg. Chem., 634, 1780-1784.

Yang, G.., \& Sevov, S. C. J. Am. (1999). Chem. Soc., 121, 8389.

Zhang, D., Yue, H., Shi, Z., Guo, M., \& Feng, S. (2006). Solid State Sci., 8, 192. 
Table 1. Details of data collection and structure refinement of ZZ-1

\begin{tabular}{|c|c|}
\hline Empirical formula & {$\left[\mathrm{C}_{10} \mathrm{H}_{24} \mathrm{~N}_{2}\right] \cdot\left[\left(\mathrm{UO}_{2}\right)_{2}\left(\mathrm{HPO}_{3}\right)_{3}\right] \cdot \mathrm{H}_{2} \mathrm{O}$} \\
\hline Formula weight & 1940.64 \\
\hline Temperature & 293(2) K \\
\hline Wavelength & 0.71073 \\
\hline Unit cell dimensions $a(\AA)$ & $18.347(4)$ \\
\hline$b(\AA)$ & $6.7341(13)$ \\
\hline$c(\AA)$ & $20.639(4)$ \\
\hline$\beta\left({ }^{\circ}\right)$ & 114.61(3) \\
\hline Volume $\left(\AA^{3}\right)$ & $2318.2(8)$ \\
\hline Z, calculated density & $2,2.78 \mathrm{~g} / \mathrm{cm}^{3}$ \\
\hline Absorption coefficient & $14.228 \mathrm{~mm}^{-1}$ \\
\hline $\mathrm{F}(000)$ & 1776 \\
\hline Crystal size & $0.2 \times 0.2 \times 0.2 \mathrm{~mm}$ \\
\hline Theta range for data collection & $2.44-25.02^{\circ}$ \\
\hline Limiting indices & $-21 \leq \mathrm{h} \leq 21,-8 \leq \mathrm{k} \leq 7,-23 \leq 1 \leq 24$ \\
\hline Reflections collected / unique & $19331 / 4054[\mathrm{R}(\mathrm{int})=0.0579]$ \\
\hline Completeness to theta $=24.99$ & $99.4 \%$ \\
\hline Absorption correction & Semi-empirical from equivalents \\
\hline Max. and min. transmission & 1.0000 and 0.2849 \\
\hline Refinement method & Full-matrix least-squares on $\mathrm{F}^{2}$ \\
\hline Goodness-of-fit on $\mathrm{F}^{2}$ & 1.018 \\
\hline Final $R$ indices $[\mathrm{I}>2 \operatorname{sigma}(\mathrm{I})]$ & $\mathrm{R} 1=0.0275, \mathrm{wR} 2=0.648$ \\
\hline $\mathrm{R}$ indices (all data) & $\mathrm{R} 1=0.0325, \mathrm{wR} 2=0.658$ \\
\hline Largest diff. peak and hole & 1.068 and $-1.352 \mathrm{e} . \AA^{-3}$ \\
\hline
\end{tabular}


Table 2. Selected bonds and angles for ZZ-1

\begin{tabular}{|c|c|c|c|}
\hline \multicolumn{4}{|c|}{ Bond lengths } \\
\hline U1-O4 & $1.772(5)$ & U2-O10 & $1.756(5)$ \\
\hline $\mathrm{U} 1-\mathrm{O} 6$ & $1.775(5)$ & U2-O11 & $1.773(5)$ \\
\hline $\mathrm{U} 1-\mathrm{O} 1^{\# 2}$ & $2.314(5)$ & $\mathrm{U} 2-\mathrm{O} 8$ & $2.272(5)$ \\
\hline U1-O5 & $2.315(5)$ & U2-O9 & $2.318(5)$ \\
\hline $\mathrm{U} 1-\mathrm{O} 2$ & $2.340(4)$ & $\mathrm{U} 2-\mathrm{O} 13^{\# 2}$ & $2.378(5)$ \\
\hline U1-07 & $2.470(5)$ & $\mathrm{U} 2-\mathrm{O} 12$ & $2.472(4)$ \\
\hline$\overline{\mathrm{U} 1-\mathrm{O} 3}$ & $2.538(5)$ & U2-O13 & $2.594(5)$ \\
\hline $\mathrm{P} 1-\mathrm{O} 1$ & $1.508(5)$ & $\mathrm{P} 2-\mathrm{O} 8$ & $1.518(5)$ \\
\hline $\mathrm{P} 1-\mathrm{O} 2$ & $1.513(5)$ & $\mathrm{P} 2-\mathrm{O} 7$ & $1.531(5)$ \\
\hline $\mathrm{P} 1-\mathrm{O} 5^{\mathrm{AI}}$ & $1.533(5)$ & $\mathrm{P} 2-\mathrm{O} 3$ & $1.533(5)$ \\
\hline$\overline{\mathrm{P} 3-\mathrm{O} 9}{ }^{\mathrm{AI}}$ & $1.509(5)$ & $\mathrm{O} 5-\mathrm{P} 1^{\mathrm{AI}}$ & $1.533(5)$ \\
\hline $\mathrm{P} 3-\mathrm{O} 12$ & $1.527(5)$ & O9-P3 ${ }^{\# 1}$ & $1.509(5)$ \\
\hline $\mathrm{P} 3-\mathrm{O} 13$ & $1.534(5)$ & $\mathrm{O} 13-\mathrm{U} 2^{\# 2}$ & $2.378(5)$ \\
\hline O1-U1 ${ }^{\# 2}$ & $2.314(5)$ & & \\
\hline \multicolumn{4}{|c|}{ Bond angles } \\
\hline O4-U1-O6 & $177.6(2)$ & O10-U2-O11 & $179.8(2)$ \\
\hline $\mathrm{O} 4-\mathrm{U} 1-\mathrm{O} 1^{\# 2}$ & $89.2(2)$ & O10-U2-O8 & $86.0(2)$ \\
\hline O6-U1-O1 ${ }^{\# 2}$ & $93.2(2)$ & O11-U2-O8 & $93.8(2)$ \\
\hline O4-U1-O5 & $90.98(19)$ & O10-U2-O9 & $92.1(2)$ \\
\hline O6-U1-O5 & $89.40(18)$ & O11-U2-O9 & $87.85(19)$ \\
\hline $\mathrm{O} 1^{\# 2}-\mathrm{U} 1-\mathrm{O} 5$ & $79.71(16)$ & O8-U2-O9 & $82.02(16)$ \\
\hline O4-U1-O2 & $91.76(19)$ & O10-U2-O13 ${ }^{\# L}$ & $88.6(2)$ \\
\hline O6-U1-O2 & $88.77(18)$ & O11-U2-O13 ${ }^{\# 2}$ & $91.6(2)$ \\
\hline $\mathrm{O} 11^{\# 2}-\mathrm{U} 1-\mathrm{O} 2$ & $78.27(16)$ & O8-U2-O13 ${ }^{\# 2}$ & $161.45(16)$ \\
\hline O5-U1-O2 & $157.76(17)$ & O9-U2-O13 ${ }^{\# 2}$ & $80.44(16)$ \\
\hline O4-U1-O7 & $86.5(2)$ & O10-U2-O12 & $92.63(19)$ \\
\hline O6-U1-O7 & $91.3(2)$ & O11-U2-O12 & $87.35(18)$ \\
\hline $\mathrm{O} 1^{{ }^{\mathrm{A}}-\mathrm{U} 1-\mathrm{O} 7}$ & $154.22(15)$ & O8-U2-O12 & $77.23(16)$ \\
\hline O5-U1-O7 & $74.97(16)$ & O9-U2-O12 & $158.34(16)$ \\
\hline $\mathrm{O} 2-\mathrm{U} 1-\mathrm{O} 7$ & $127.22(16)$ & $\mathrm{O} 13^{\# 2}-\mathrm{U} 2-\mathrm{O} 12$ & $120.80(15)$ \\
\hline $\mathrm{O} 4-\mathrm{U} 1-\mathrm{O} 3$ & $91.88(19)$ & O10-U2-O13 & $83.1(2)$ \\
\hline O6-U1-O3 & $86.15(19)$ & O11-U2-O13 & $97.0(2)$ \\
\hline $\mathrm{O} 1^{\# 2}-\mathrm{U} 1-\mathrm{O} 3$ & $147.06(15)$ & O8-U2-O13 & $131.69(16)$ \\
\hline O5-U1-O3 & $133.16(16)$ & O9-U2-O13 & $145.11(15)$ \\
\hline $\mathrm{O} 2-\mathrm{U} 1-\mathrm{O} 3$ & $68.79(16)$ & $\mathrm{O} 13^{\# 2}-\mathrm{U} 2-\mathrm{O} 13$ & $64.95(18)$ \\
\hline O7-U1-O3 & $58.58(15)$ & O12-U2-O13 & $56.53(14)$ \\
\hline $\mathrm{O} 1-\mathrm{P} 1-\mathrm{O} 2$ & $112.0(3)$ & O8-P2-O7 & $114.6(3)$ \\
\hline $\mathrm{O} 1-\mathrm{P} 1-\mathrm{O} 5^{\# 1}$ & $113.4(3)$ & $\mathrm{O} 8-\mathrm{P} 2-\mathrm{O} 3$ & $114.3(3)$ \\
\hline $\mathrm{O} 2-\mathrm{P} 1-\mathrm{O} 5^{\mathrm{H1}}$ & 109.4(3) & $\mathrm{O} 7-\mathrm{P} 2-\mathrm{O} 3$ & $106.2(3)$ \\
\hline $\mathrm{O} 9^{\mathrm{A1}}-\mathrm{P} 3-\mathrm{O} 12$ & $115.2(3)$ & $\mathrm{P} 1-\mathrm{O} 1-\mathrm{U} 1^{\# 2}$ & $138.6(3)$ \\
\hline $\mathrm{O} 9^{\mathrm{A1}}-\mathrm{P} 3-\mathrm{O} 13$ & $113.2(3)$ & $\mathrm{P} 1-\mathrm{O} 2-\mathrm{U} 1$ & $136.9(3)$ \\
\hline O12-P3-O13 & $103.3(3)$ & P2-O3-U1 & $96.0(2)$ \\
\hline $\mathrm{P} 1^{\mathrm{A1}}-\mathrm{O} 5-\mathrm{U} 1$ & 141.4(3) & P3-O12-U2 & $101.7(2)$ \\
\hline P2-O7-U1 & $98.8(2)$ & $\mathrm{P} 3-\mathrm{O} 13-\mathrm{U} 2^{\# 2}$ & $148.5(3)$ \\
\hline P2-O8-U2 & $147.5(3)$ & P3-O13-U2 & $96.4(2)$ \\
\hline $\mathrm{P}^{\mathrm{H1}}-\mathrm{O} 9-\mathrm{U} 2$ & $144.8(3)$ & $\mathrm{U} 2^{\# 2}-\mathrm{O} 13-\mathrm{U} 2$ & $115.05(17)$ \\
\hline
\end{tabular}

Symmetry transformation used to generate equivalent atoms: $1:-\mathrm{x}+1,-\mathrm{y},-\mathrm{z}+1 ; 2: \mathrm{x}, \mathrm{y}+1, \mathrm{z}$. 


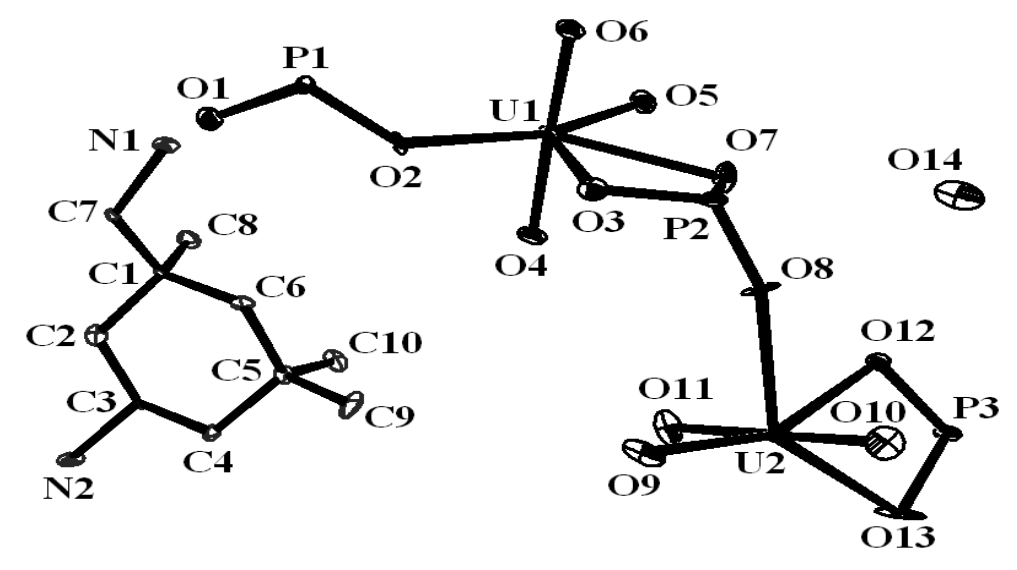

A

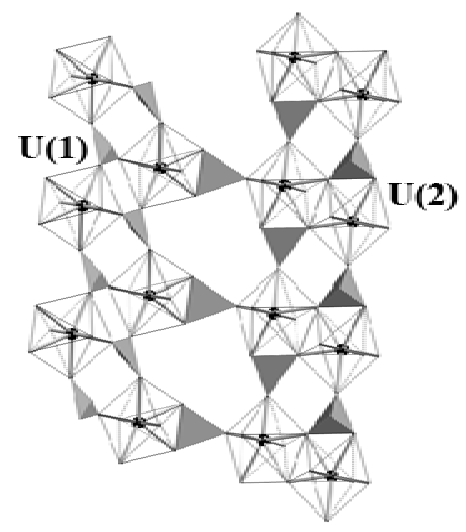

B
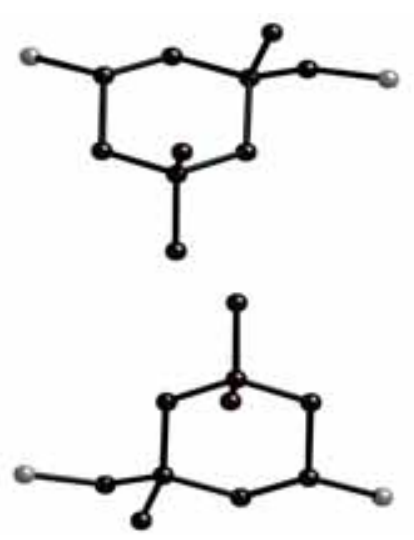

C

Figure 1. ORTEP plot of ZZ-1 showing the labeling scheme(A);Polyhedral representations of the two types of four-ring chains in $\mathrm{ZZ}-1(\mathrm{~B}): \mathrm{HPO}_{3}$ linked $\mathrm{U}(1) \mathrm{O}_{7}$ monomer and $\mathrm{HPO}_{3}$ connected $\mathrm{U}(2)_{2} \mathrm{O}_{12}$, and $(\mathrm{C})$ ball and stick model of the IPDA cations in cis- conformation. 


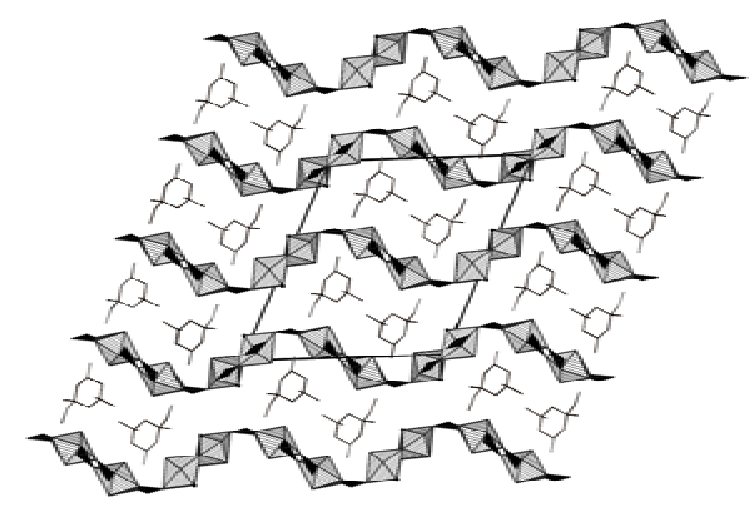

A

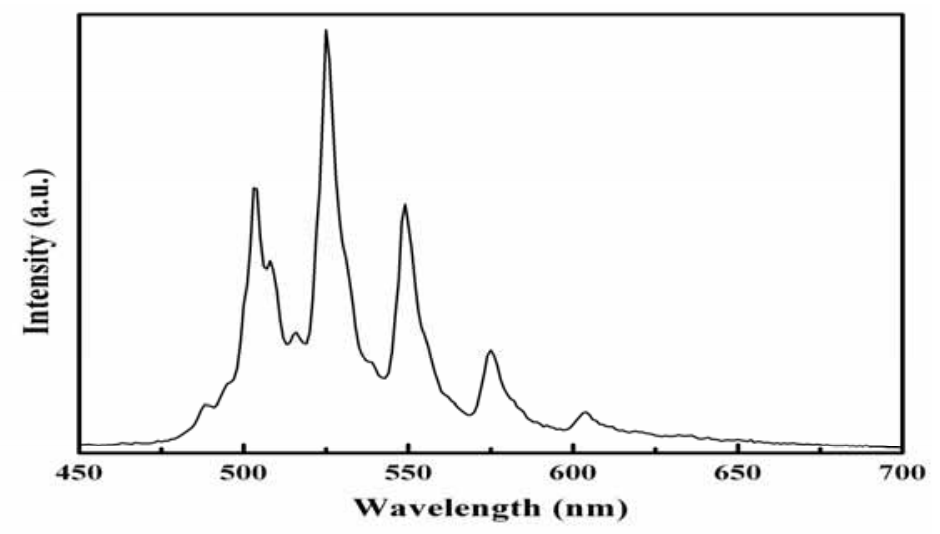

B

Figure 2. The layer structure with the intercalated $\mathrm{H}_{2}$ IPDA cations of ZZ-1 viewed along the b direction 


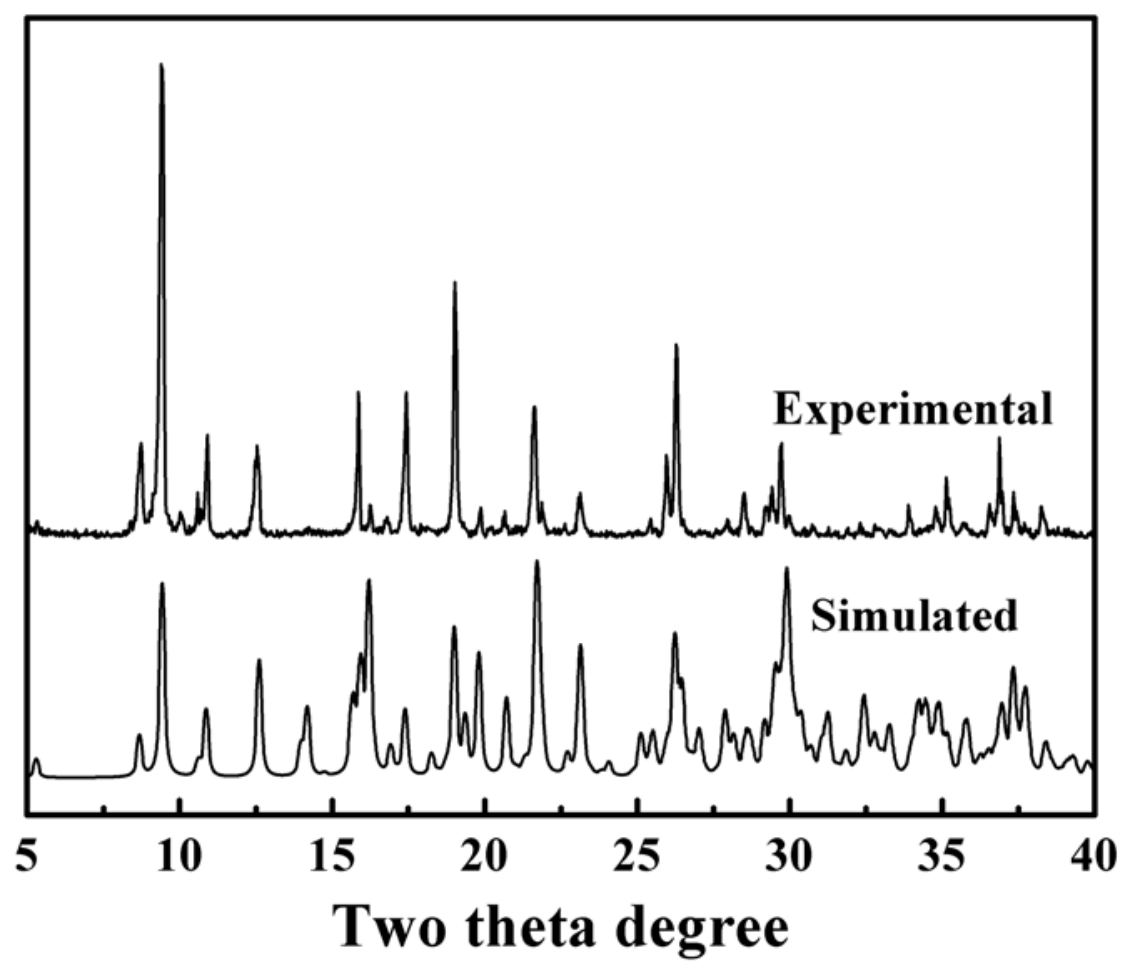

Figure 3. Simulated and experimental XRD patterns for ZZ-1

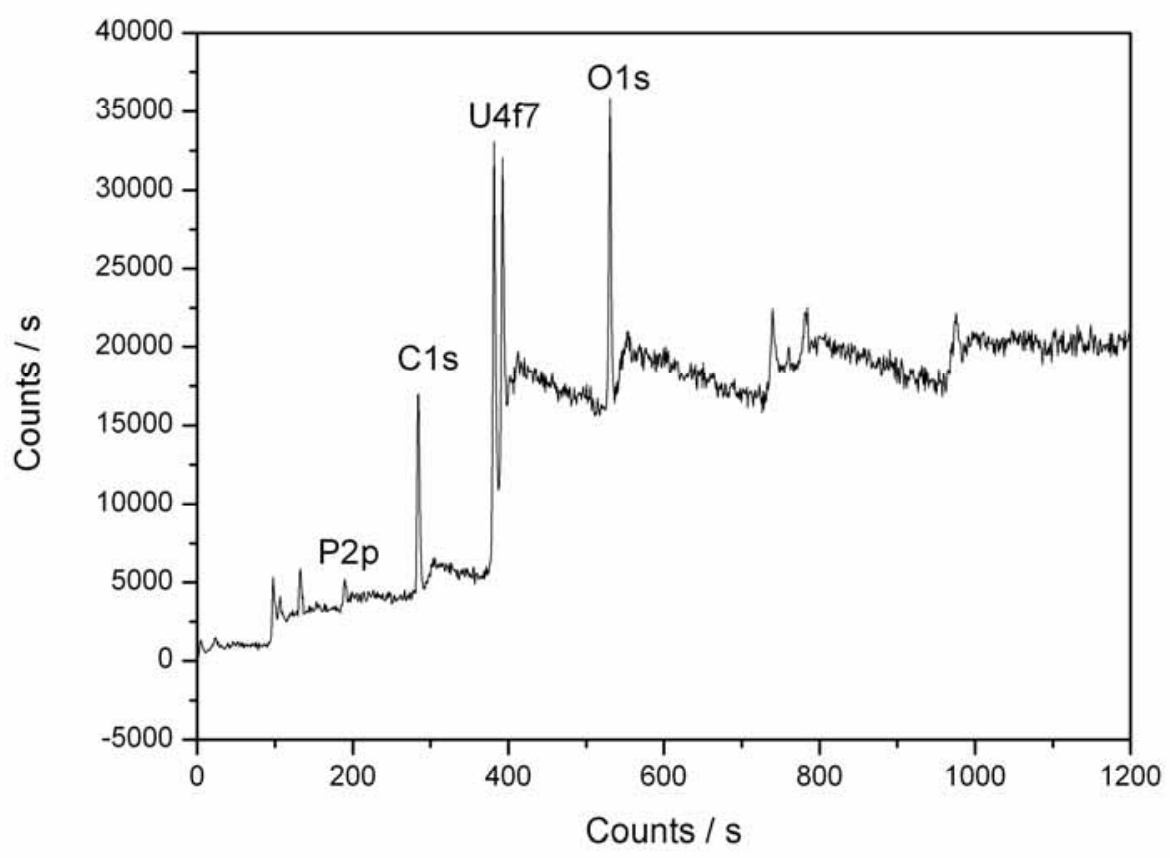

Figure 4. XPS curve for ZZ-1 